hearing disorders are a significant complication of fetal alcohol exposure, probably contributing to the speech and language and learning disabilities commonly associated with the syndrome. (Church MW, Gerkin KP. Hearing disorders in children with fetal alcohol syndrome: findings from case reports. Pediatrics August 1988; 82:147 $-154)$.

COMMENT. As a cause of mental retardation, fetal alcohol syndrome is as prevalent as Down's syndrome and occurs in at least one in 1000 births. Postmortem examination of the brain reveals extensive developmental defects, mostly the consequence of migration anomalies. Heterotopias, lissencephaly, and agenesis of the corpus callosum have been described. Migration anomalies may involve the brain stem as well as the cerebrum, thus accounting for the sensorineural deafness noted in the above study.

\title{
VALPROATE TOXICITY
}

Gastritis and erosion of the gastric mucosa is reported in 10 children who presented with feeding difficulties after long-term treatment with divalproex sodium (Depakote) and valproic acid (Depakene) at the Departments of Neurology, Pediatrics, and Pediatric Surgery, University of Oklahoma Health Sciences Center, Oklahoma City. Anorexia and refusal to eat were complicated by vomiting in 8 patients, abdominal pain in 5, weight loss in 3, and diarrhea in 2 . Endoscopy confirmed erosive gastritis. Treatment with oral antacids (Malox) and cimetidine resulted in clinical improvement. (Marks WA et al. Gastritis with valproate therapy. Arch Neurol August 1988; $\underline{45}: 903-905$ ).

COMMENT. Gastrointestinal disturbances are common at the initiation of valproate therapy but have not previously been reported following its long term use. The diagnosis of gastritis in mentally retarded patients with epilepsy may be difficult, and delay may lead to gastric erosion, resulting in malnutrition, weight loss, and dehydration. If liver and pancreas disease has been excluded as a side-effect, the treatment of valproate-induced gastritis includes antacids and an alteration of anticonvulsant regimen in some cases.

\section{NEONATAL SEIZURES}

\section{INFANTILE SPASMS, HYPSARRHYTHMIA, AND ADRENOLEUKODYSTROPHY (ALD)}

An 8 1/2 month-old girl with seizures beginning at 5 days, hypsarrhythmia in the EEG, severe retardation, and a clinical diagnosis of infantile spasms was discovered to have biochemical and pathological features of adrenoleukodystrophy, as reported from the John F. Kennedy Institute, Johns Hopkins University, Baltimore, Maryland. Laboratory studies showed elevated plasma levels of very long chain fatty acids, and postmortem examination at 14 months revealed cerebral destructive lesions and adrenal cortex atrophy. Seizure frequency had diminished initially with prednisone 
$20 \mathrm{mg} / \mathrm{daily}$, but improvement was not maintained. The biochemical changes resembled the abnormalities observed in X-linked ALD and differed from those in the neonatal form. Comparison with other known peroxisomal disorders suggested a unique example of this category of disease. (Naidu $S$ et al. Neonatal seizures and retardation in a girl with biochemical features of $\mathrm{X}-\mathrm{linked}$ adrenoleukodystrophy: a possible new peroxisomal disease entity.

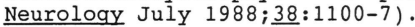

COMMENT. For an excellent review of the various entities now classified as generalized peroxisomal disorders, please refer to Naidu, Moser, Moser. Pediatr Neurol 1988; 4 :5 (reviewed in Ped Neur Briefs 1988;2:30). Immunopathological factors have been postulated in the pathogenesis of CNS lesions in X-linked adrenoleukodystrophy. Cyclophosphamide administered to 4 boys between 6 and 11 years of age with proven ALD failed to slow the rate of neurological progression. (Naidu $S$ et al. Arch Neurol

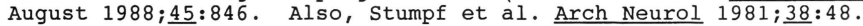

\section{OUTCOME OF NEONATAL CONVULSIONS}

The risk factors, causes, and prognosis of convulsions in 156 neonates are reviewed at the Mater Misericordiae Mothers Hospital, South Brisbane, Queensland, Australia. The incidence of early neonatal convulsions was $3 / 1000$ live births. Compared to infants who did not convulse, the leading risk factors for convulsions were prematurity, intra-uterine growth retardation, low 5 min Apgar score, pre-eclampsia, antepartum hemorrhage, twin pregnancy, and breech presentation. The cause was hypoxic-ischemic encephalopathy (HIE) in $40 \%$, intracranial hemorrhage (30\%), metabolic (12\%), infection (8\%), malformation (3\%), misc $(7 \%)$. Mortality (31\%) was related to etiology: $57 \%$ for intracranial hemorrhage, $33 \%$ infection, $27 \%$ HIE. of the 107 infants who survived, long-term disability occurred in 43\%; severe in 25 infants, moderate in 8 and mild in 10 . The highest mortality and morbidity are associated with prolonged convulsions, tonic and multifocal clonic convulsions, convulsions due to asphyxia and intracranial hemorrhage, and an abnormal neurologic examination at discharge. (Tudehope DI et al. Clinical spectrum and outcome of neonatal convulsions. Aust Paediatr J August 1988; 24 :249-253).

COMMENT. The outcome of neonatal convulsions in this study is similar to that reported in a Dublin Collaborative Study of neonatal asphyxial seizures in which $43 \%$ had a poor outcome (Curtis PD et al. Arch Dis Childh September 1988;63:1065-8). In the Dublin study, asphyxial seizures occurring within 48 hours of birth in $0.87 / 1000$ live births were correlated with antenatal complications, primiparity, and prolonged pregnancy. The incidence of seizures ranged from $0.55-1.2 / 1000$ in the 3 participating maternity hospitals, reflecting differences in management policies in regard to frequency of cesarean section, induced labor, and forceps delivery. The mortality rate was $18 \%$ and of those who survived, 288 were handicapped at 1 year. Outcome was correlated with the infants' feeding habits at 1-2 